\title{
Adenosine potentiates the therapeutic effects of neural stem cells expressing cytosine deaminase against metastatic brain tumors
}

\author{
WONYOUNG KANG ${ }^{1-3 *}$, HO JUN SEOL ${ }^{1 *}$, DONG-HO SEONG ${ }^{4}$, JANDI KIM ${ }^{4}$, \\ YONGHYUN KIM ${ }^{6}$, SEUNG U. KIM ${ }^{7,8}$, DO-HYUN NAM ${ }^{1-3}$ and KYEUNG MIN JOO ${ }^{3-5}$
}

${ }^{1}$ Department of Neurosurgery, ${ }^{2}$ Samsung Biomedical Research Institute, ${ }^{3}$ Samsung Advanced Institute for Health Sciences and Technology, Samsung Medical Center, Sungkyunkwan University School of Medicine, Seoul; ${ }^{4}$ Department of Anatomy and ${ }^{5}$ Center for Molecular Medicine, Samsung Biomedical Research Institute, Sungkyunkwan University School of Medicine, Suwon, Republic of Korea; ${ }^{6}$ Department of Chemical and Biological Engineering, The University of Alabama, Tuscaloosa, AL, USA; ${ }^{7}$ Division of Neurology, Department of Medicine, University of British Columbia,Vancouver, BC, Canada;

${ }^{8}$ Medical Research Institute, Chung-Ang University College of Medicine, Seoul, Republic of Korea

Received November 13, 2012; Accepted January 7, 2013

DOI: $10.3892 / o r .2013 .2584$

\begin{abstract}
Tumor-tropic properties of neural stem cells (NSCs) provide a novel approach with which to deliver targeting therapeutic genes to brain tumors. Previously, we developed a therapeutic strategy against metastatic brain tumors using a human NSC line (F3) expressing cytosine deaminase (F3. CD). F3.CD converts systemically administered 5-fluorocytosine (5-FC), a blood-brain barrier permeable nontoxic prodrug, into the anticancer agent 5-fluorouracil (5-FU). In this study, we potentiated a therapeutic strategy of treatment with nucleosides in order to chemically facilitate the endogenous conversion of 5-FU to its toxic metabolite 5-FU ribonucleoside (5-FUR). In vitro, 5-FUR showed superior cytotoxic activity against MDA-MB-435 cancer cells when compared to 5-FU. Although adenosine had little cytotoxic activity, the addition of adenosine significantly potentiated the in vitro cytotoxicity of 5-FU. When MDA-MB-435 cells were co-cultured with F3.CD cells, F3.CD cells and 5-FC inhibited the growth of MDA-MB-435 cells more significantly
\end{abstract}

Correspondence to: Professor Do-Hyun Nam, Department of Neurosurgery, Samsung Medical Center and Samsung Biomedical Research Institute, Sungkyunkwan University School of Medicine, Samsung Cancer Center, 50 Irwon-Dong, Gangnam-gu, Seoul 135-710, Republic of Korea

E-mail: nsnam@skku.edu

Professor Kyeung Min Joo, Department of Anatomy, Center for Molecular Medicine, Samsung Biomedical Research Institute, Sungkyunkwan University School of Medicine, Suwon 440-746, Republic of Korea

E-mail:kmjoo@skku.edu

${ }^{*}$ Contributed equally

Key words: neural stem cell, cytosine deaminase, adenosine, 5-fluorocytosine, 5-fluorouracil ribonucleoside, metastatic brain tumor in the presence of adenosine. Facilitated 5-FUR production by F3.CD was confirmed by an HPLC analysis of the conditioned media derived from F3.CD cells treated with 5-FC and adenosine. In vivo systemic adenosine treatment also significantly potentiated the therapeutic effects of F3.CD cells and 5-FC in an MDA-MB-435 metastatic brain tumor model. Simple adenosine addition improved the antitumor activity of the NSCs carrying the therapeutic gene. Our results demonstrated an increased therapeutic potential, and thereby, clinical applicability of NSC-based gene therapy.

\section{Introduction}

Advancements in the diagnosis and treatment of primary cancers have paradoxically led to an increased incidence of metastatic brain tumors (MBTs) (1) that have an unfavorable clinical prognosis (2). Since patients with metastasis typically have multiple brain lesions, local treatment modalities such as surgery and radiation therapy are impractical. Furthermore, chemotherapeutic agents typically cannot penetrate through the blood-brain barrier (BBB), making systemic chemotherapy ineffective (3). Palliative whole-brain irradiation or stereotactic radiosurgery is currently the sole viable option for MBTs.

The tumor-tropic properties of neural stem cells (NSCs) provide a novel approach that can overcome the aforementioned challenges in developing chemotherapy regimens for MBTs (4). NSCs can be genetically engineered to carry therapeutic genes to tumor lesions. For example, cytosine deaminase (CD), which converts 5-fluorocytosine $(5-\mathrm{FC})$ to 5-fluorouracil (5-FU), can be engineered into NSCs to specifically target the multiple lesion sites of MBTs. This is significant since 5-FU, although effective, cannot penetrate the $\mathrm{BBB}$ and have toxic effects $(3,5-8)$, while $5-\mathrm{FC}$ is BBB-permeable and nontoxic allowing systemic administration. We previously used an immortalized human NSC line expressing CD and showed its significant therapeutic effects against brain tumors $(5,7)$.

In order to ensure therapeutic effects, there must be a high local concentration of 5-FU. However, 5-FU itself does not act 
as a strong cytotoxic compound until it is converted to 5-FU ribonucleoside (5-FUR) by endogenous enzymes $(9,10)$. As one strategy to enhance this conversion, a previous report showed that the expression of uracil phosphoribosyl transferase in small-cell lung cancers led to superior therapeutic effects (11). For therapeutic purposes, however, such genetic manipulation of cancer cells is not plausible. As an alternative strategy, the level of ribose-1-phosphate in cancer cells can be increased by supplementing excess levels of ribonucleosides to drive the endogenous biochemical reaction of 5-FU to 5-FUR (Fig. 2). Therefore in this study, we explored the effects of systemic administration of ribonucleosides, in addition to the administration of 5-FU pro-drug 5-FC, to potentiate the therapeutic effects of genetically engineered NSCs for the treatment of MBTs in in vitro and in vivo models.

\section{Materials and methods}

Cell culture. Immortalized human fetal NSCs (F3), F3 NSCs expressing Escherichia coli CD (F3.CD), and a human breast cancer cell line MDA-MB-435 (American Type Culture Collection, Manassas, VA) were cultured in Dulbecco's modified Eagle's medium (DMEM) supplemented with $10 \%$ fetal bovine serum, $2 \mathrm{mM}$ L-glutamine, $100 \mathrm{U} / \mathrm{ml}$ penicillin, $100 \mu \mathrm{g} /$ $\mathrm{ml}$ streptomycin and $0.25 \mu \mathrm{g} / \mathrm{ml}$ amphotericin B (Invitrogen).

Genetic engineering of F3.CD. Preparation of the F3.CD from the parental F3 line was previously described $(5,12,13)$. Briefly, an expression plasmid encoding $C D$ was constructed using the retroviral pBABE-puro backbone and 1.5-kDa CD cDNA. Vectors were packaged by co-transfecting PA317 cells with the expression plasmid and the MV12 envelope-coding plasmid. The resulting supernatant was used for multiple infections of F3 cells. Transduced F3.CD cells were selected with $3 \mu \mathrm{g} / \mathrm{ml}$ puromycin (Invitrogen) over four weeks.

Cytotoxic activities of 5-FU and 5-FUR. To confirm the differential cytotoxic activity of 5-FU and 5-FUR, $1 \times 10^{4}$ MDA-MB-435 cells were plated in 96-well plates. Twenty-four hours after seeding, 5-FU or 5-FUR (Sigma) (0, 0.05, 0.5, 5, 50 or $500 \mathrm{nM}$ ) was applied for $48 \mathrm{~h}$. The status of the cells was analyzed using a microscope, and their viability was determined with a colorimetric assay (Cell Counting Kit-8; Dojindo Molecular Technologies).

In vitro effects of ribonucleoside supplementation.

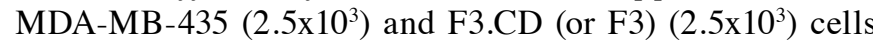
were co-cultured in 96-well plates for 24 h. 5-FU (or 5-FC) $(0.5 \mathrm{mM})$ and $0.2 \mathrm{mM}$ ribonucleoside (adenosine, guanosine or uridine) was added. After a 48-h treatment, viability was measured as previously described. To confirm the enhanced production of 5-FUR, $1.5 \times 10^{6} \mathrm{~F} 3$.CD cells were suspended in $2 \mathrm{ml}$ PBS with $2 \mathrm{mM} 5$-FC with or without $10 \mathrm{mM}$ adenosine at $37^{\circ} \mathrm{C}$ for $48 \mathrm{~h}$. The resulting conditioned media were collected, mixed with an equal volume of methanol, and filtered by sterilized PVDF filters. The filtrates were analyzed by HPLC (Younglin; Solvent Delivery Pump SP930D, UV/730D absorbance detector, rheodyne injector) using Capcell Pak $\mathrm{C}_{18}$ column (type UG120, $5 \mu \mathrm{m}$, size $4.6 \mathrm{~mm}$ ID x $250 \mathrm{~mm}$; Shiseido Co., $1 \mathrm{ml} / \mathrm{min}$ flow rate, $265 \mathrm{~nm}$ detection).
In vivo effects of systemic adenosine administration on the F3.CD and 5-FC treatment against MBTs. The animal experiments were approved by the Review Board of Samsung Biomedical Research Institute (Seoul, Korea). MDA-MB-435 cells $\left(1 \times 10^{5}\right)$ in $10 \mu \mathrm{l}$ HBSS were directly implanted into the brains of anesthetized BALB/c-nu mice (6-weeks old) using a rodent stereotactic frame [co-ordinates: anterior/posterior (AP) $+1.0 \mathrm{~mm}$, medial/lateral $(\mathrm{ML})+1.7 \mathrm{~mm}$, dorsal/ventral (DV) $-3.2 \mathrm{~mm}$, depth from the dura matter $2 \mathrm{~mm}$ ]. Thirteen and twenty days after MDA-MB-435 cell implantation, animals were subjected to contralateral injection $(\mathrm{AP}+1.0 \mathrm{~mm}, \mathrm{ML}$ $-1.7 \mathrm{~mm}$, DV $-3.2 \mathrm{~mm}$ ) of $5 \mu \mathrm{l} \mathrm{HBSS}$ (Group I/II, $\mathrm{n}=10$ for each group) or F3.CD cells $\left(1 \times 10^{5}\right)$ in $5 \mu 1 \mathrm{HBSS}$ (Group III/IV, $\mathrm{n}=10$ for each group). The Group II/IV and Group III/IV received intraperitoneal (i.p.) injection of adenosine $(200 \mathrm{mg} / \mathrm{kg}$ in $200 \mu \mathrm{l}$ PBS) and 5-FC (500 mg/kg in $200 \mu 1$ normal saline), respectively, every day for 5 days after each contralateral injection. Two days after the last i.p. injection, brains were removed and cut into 4- to 6-mm slices. For tumor volume measurement (largest width ${ }^{2} \mathrm{x}$ largest length $\mathrm{x} 0.5$ ), the brain slices were fixed in $10 \%$ formalin/PBS, embedded in paraffin, sectioned into $4-\mu \mathrm{m}$ coronal sections, and stained with hematoxylin and eosin (H\&E). A rabbit anti-CD polyclonal antibody (1:1,000; Dr K.S. Aboody, City of Hope Medical Center, Duarte, CA, USA) was used for immunostaining.

Statistics. Statistical comparisons were performed using the Student's t-test. Survival analysis was performed using the Kaplan-Meier and the log-rank tests. P-values $<0.05$ were considered to indicate a statistically significant result.

\section{Results}

Superior cytotoxic activity of 5-FUR against MDA-MB-435 cells. To confirm the differential cytotoxic activity of 5-FU and 5-FUR, MDA-MB-435 cells were treated with 5-FU or 5 -FUR $(0,0.05,0.5,5,50$ or $500 \mathrm{nM})$ for $48 \mathrm{~h}$. When cell viability was determined with a colorimetric assay, 5-FUR showed significantly higher cytotoxic effects than 5-FU at all tested doses (Fig. 3A). These results correlate well with a prior report, which showed that the cytotoxic effect of 5-FU is mediated by its toxic metabolite 5-FUR (10).

In vitro effects of ribonucleoside supplementation on the cytotoxicity of 5-FU. Sufficient ribose-1-phosphate supplementation facilitates the conversion reaction of 5-FU to 5-FUR. When MDA-MB-435 or F3.CD cells were treated with 0.02 , 0.2 or $2 \mathrm{mM}$ ribonucleoside (adenosine, guanosine, or uridine) for $48 \mathrm{~h}$, adenosine and guanosine showed toxicity to the cells at $2 \mathrm{mM}$ (Fig. 1). In doses $<2 \mathrm{mM}$, cells tolerated the addition of all ribonucleosides (Fig. 1). Sensitivity of F3.CD, F3, and MDA-MB-435 cells to 5-FC and 5-FU was tested. 5-FU decreased the viability of all tested cells, but 5-FC only decreased the viability of the F3.CD cells (data not shown). Viability of F3 and MDA-MB-435 cells was not affected by 5-FC addition up to $0.5 \mathrm{mM}$ (data not shown). Adenosine was selected for further experiments since it exerted the greatest reduction in viability (Fig. 1). The combination of 5-FU and adenosine increased the cytotoxic effect in both MDA-MB-435 and F3.CD cells (Fig. 3B and C), suggesting that adenosine 

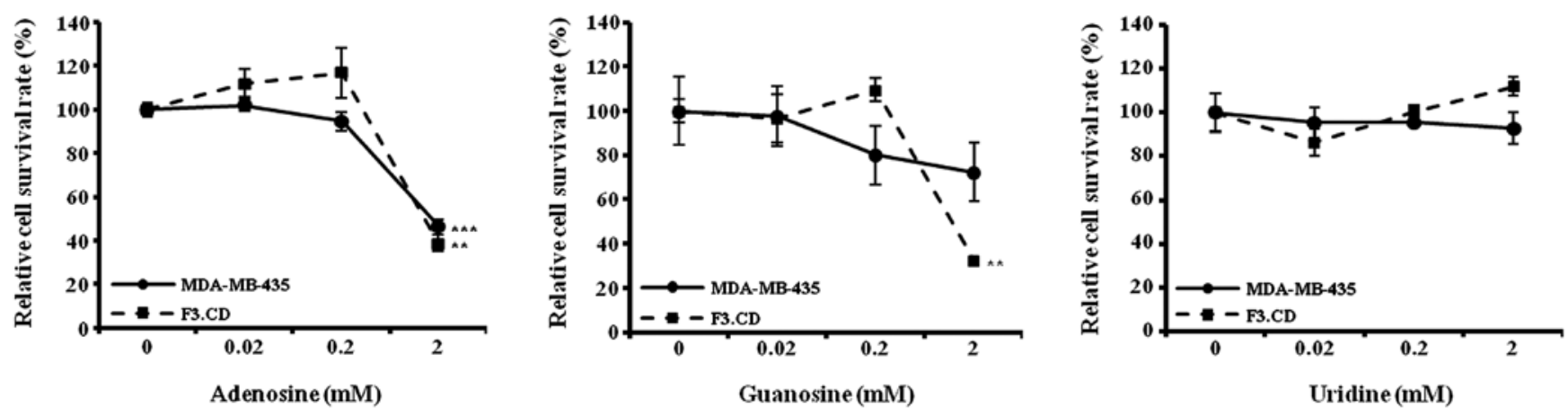

Figure 1. Effects of nucleoside addition on the viability of MDA-MB-435 and F3.CD cells. The effects of increasing concentrations of ribonucleosides on the in vitro survival of MDA-MB- 435 or F3.CD cells were evaluated. ${ }^{* *} \mathrm{P}<0.01 ;{ }^{* * *} \mathrm{P}<0.001$ vs. no treatment.

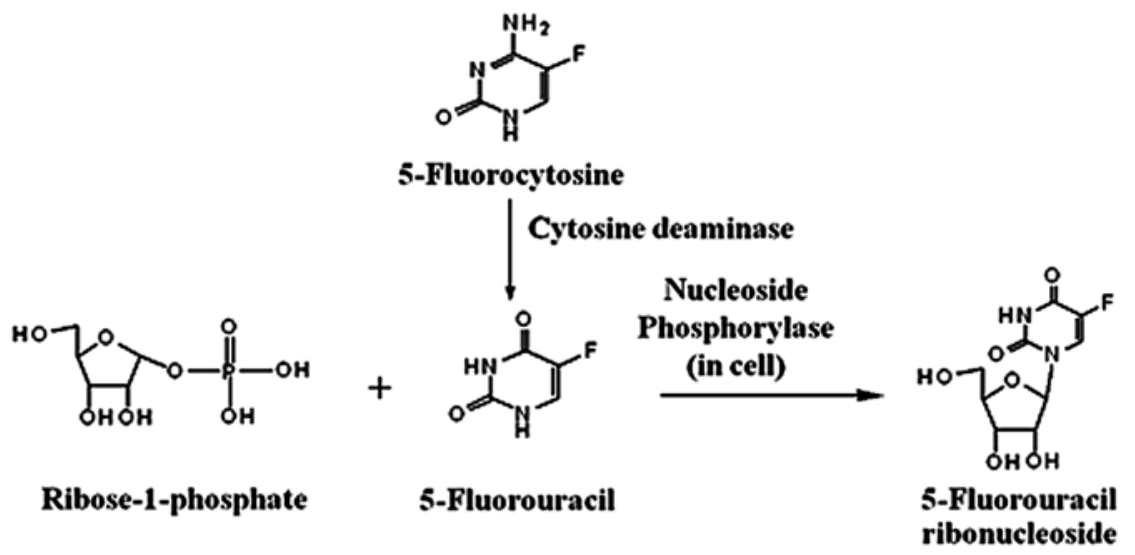

Figure 2. Proposed cytotoxic mechanism of ribonucleoside supplementation. Ribose-1-phosphate enhances the production of 5-fluorouracil ribonucleoside (5-FUR), the cytotoxic metabolite of 5-fluorouracil (5-FU).

supplementation potentiated the therapeutic effects of F3.CD and 5-FC. To confirm the facilitated production of 5-FUR, the 48-h conditioned media from F3.CD cells with $2 \mathrm{mM}$ 5-FC and with or without $10 \mathrm{mM}$ adenosine were analyzed by HPLC. 5-FU was detected in both groups, whereas 5-FUR was found only in the adenosine-treated group (Fig. 3D). These results indicate that F3.CD cells have the ability to convert 5-FC to 5-FU and that the production of toxic 5-FUR was facilitated by the supplementation of adenosine.

In vitro effect of adenosine supplementation on the bystander

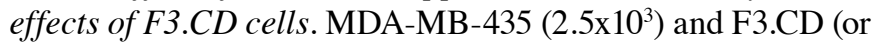
F3) cells $\left(2.5 \times 10^{3}\right)$ were co-cultured for 24 h. 5-FU (or 5-FC) $(0.5 \mathrm{mM})$ and adenosine $(0,125,250$ or $500 \mathrm{nM})$ were added for 48 h. When MDA-MB-435 and F3.CD cells were co-cultured, the cytotoxic effect of 5-FU was elevated dose-dependently in regards to the adenosine addition $(\mathrm{P}<0.001$, Fig. 4A), whereas adenosine treatment without 5 -FU showed only mild toxicity at $500 \mathrm{nM}$ (Fig. 4A). In vitro cytotoxic effects of 5-FC and/or adenosine were not observed in the MDA-MB-435 and F3 co-culture (Fig. 4B). However, addition of adenosine increased the bystander cytotoxicity of F3.CD and 5-FC on MDA-MB-435 cells dose-dependently $(\mathrm{P}<0.01$, Fig. 4B). Therefore, the adenosine treatment improved the therapeutic activities of the NSCs expressing CD.
In vivo effects of adenosine supplementation on the F3.CD and 5-FC treatment against brain metastatic tumors. MDA-MB-435 cells $\left(1 \times 10^{5}\right)$ were directly implanted into the brains of BALB/c-nu mice (Fig. 5A). Thirteen and twenty days after the tumor cell implantation, animals were subjected to contralateral injection of $5 \mu 1$ HBSS (Group I/II, n=10 for each group) or $1 \times 10^{5} \mathrm{~F} 3$.CD cells in $5 \mu \mathrm{HBSS}$ (Group III/IV, $\mathrm{n}=10$ for each group) (Fig. 5A). Group II/IV and Group III/IV received i.p. injection of adenosine $(200 \mathrm{mg} / \mathrm{kg})$ and $5-\mathrm{FC}$ $(500 \mathrm{mg} / \mathrm{kg})$, respectively, every day for 5 days after each contralateral injection (Fig. 5A). Two days after the last i.p. injection, tumor volumes and migration of implanted F3.CD cells were measured (Fig. 5A). A large number of CD-immunoreactive F3.CD cells were identified in the tumor bed and at the tumor normal parenchyma interface. Some migrating F3.CD cells were also found in the corpus callosum (Fig. 5B), confirming the tumor-tropic activity of F3.CD cells. Histological analysis showed significantly $(\mathrm{P}<0.05)$ reduced tumor volumes in the brains of the F3.CD and 5-FC-treated animals (Group III) compared with the control groups [Group I, 18.3 $\pm 5.4 \mathrm{~mm}^{3}$ (mean $\pm \mathrm{SE}$ ); Group II, $17.3 \pm 4.9 \mathrm{~mm}^{3}$; Group III, $6.9 \pm 1.6 \mathrm{~mm}^{3}$ ] (Fig. 5C and D). Systemic administration of adenosine (Group II) had no significant effects on the tumor volume, compared with the control group (Group I) (Fig. 5C and D). Group IV, which 

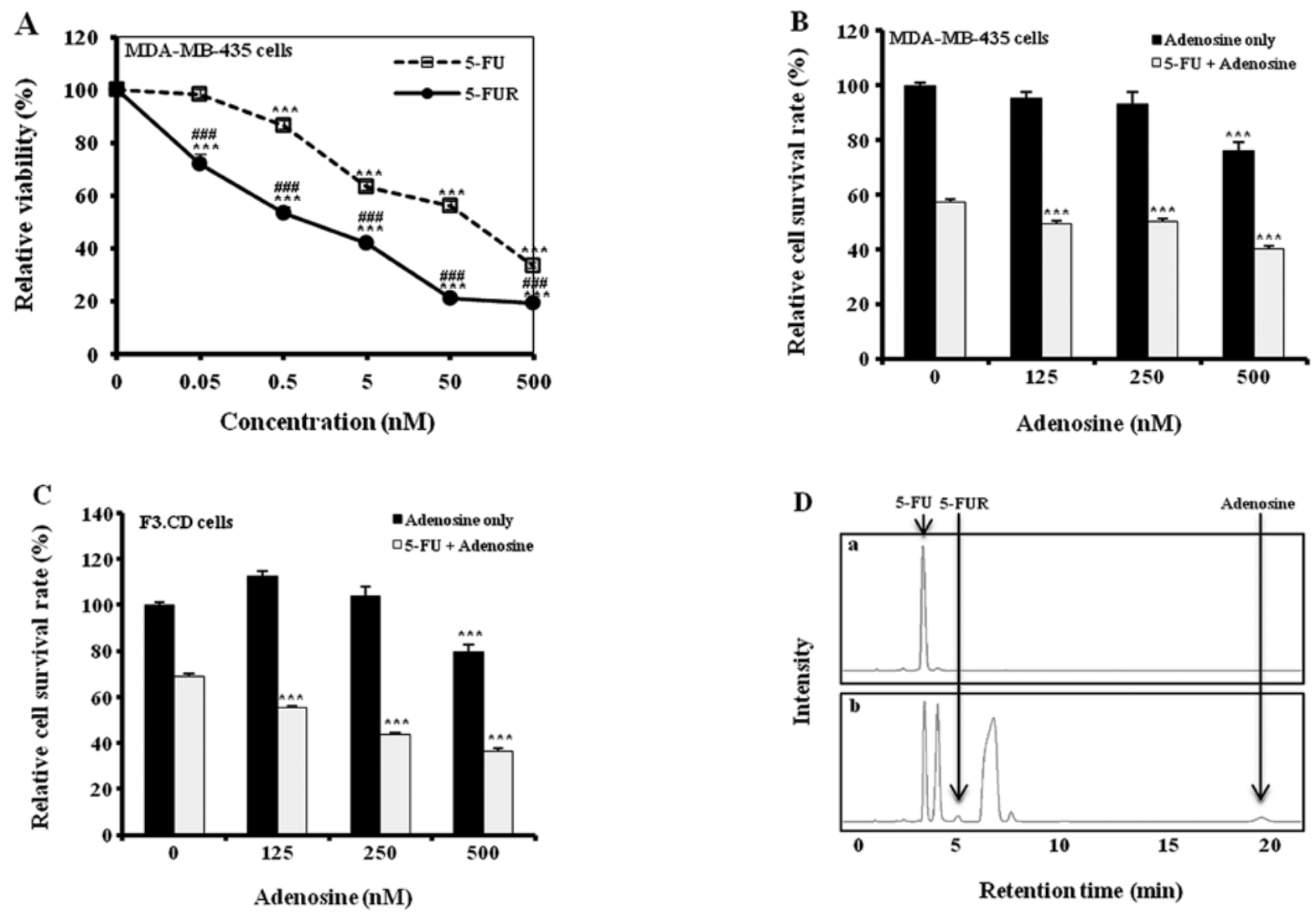

Figure 3. In vitro effects of ribonucleoside supplementation on the cytotoxic effect of 5-FU. (A) Cytotoxic effects of 5-FU and 5-FUR were compared using MDA-MB-435 cells. Adenosine supplementation potentiated the cytotoxic effect of 5-FU against (B) MDA-MB-435 and (C) F3.CD cells. 5-FU, 5-fluorouracil; 5-FUR, 5-FU ribonucleoside. Bars, mean; error bars, SE; ${ }^{* * *} \mathrm{P}<0.001$ vs. no treatment of each drug; ${ }^{\# \# " ~} \mathrm{P}<0.001$ vs. FU at each dose. (D) Chromatograms of high performance liquid chromatography (HPLC). Conditioned media derived from F3.CD cells treated with (a) 5-FC or (b) 5-FC + adenosine were analyzed by HPLC.
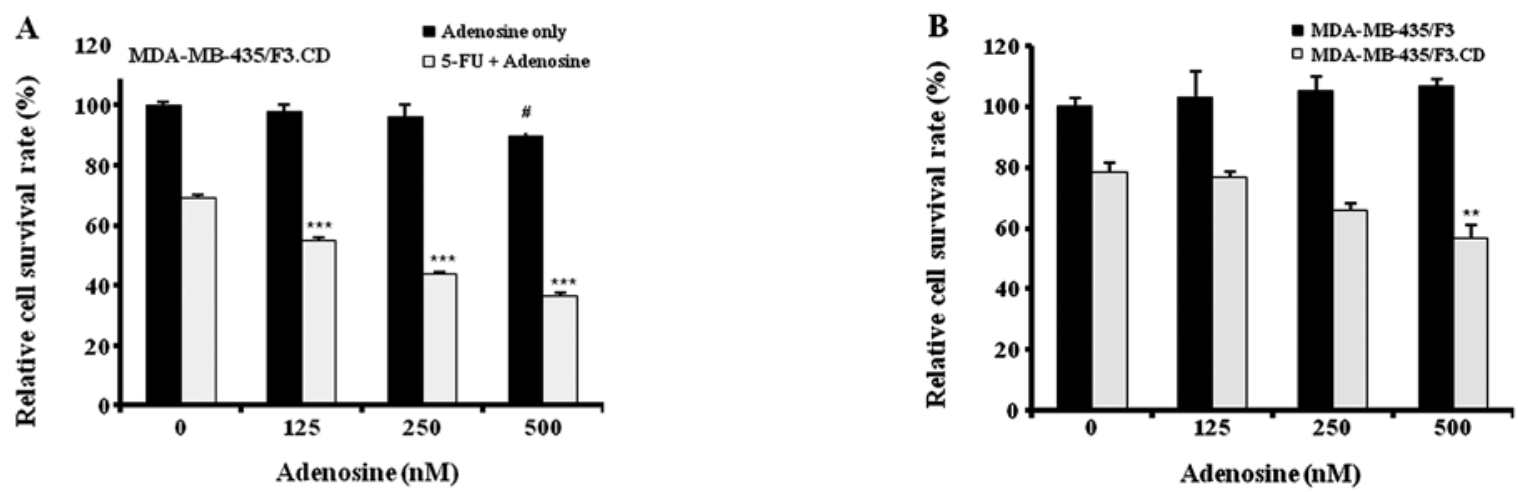

Figure 4. Adenosine increased the bystander effects of F3.CD cells. (A) When 5-FU and/or adenosine was added to the MDA-MB-435 and F3.CD co-culture, cytotoxicity of 5-FU was significantly increased by the supplementation of adenosine. (B) Effects of adenosine on cytotoxicity of 5-FC were determined in MDA-MB-435 and F3.CD (or F3) co-culture. 5-FU, 5-fluorouracil. Column, mean; bar, $\mathrm{SE} ;{ }^{* *} \mathrm{P}<0.01 ;{ }^{* * *} \mathrm{P}<0.001 \mathrm{vs} .0 \mathrm{nM}$ adenosine.

underwent F3.CD + 5-FC treatment and additional systemic adenosine supplementation, showed a further decrease in the tumor volume $\left(3.5 \pm 0.9 \mathrm{~mm}^{3}\right)$ at a statistically significant level compared with that of Group III (Fig. 5C and D).

\section{Discussion}

Recent studies have found that immortalized and genetically engineered NSCs display tumor-tropic activities that may be exploited for tumor-specific gene therapy for various types of tumors including MBTs $(3,5,7,8,14)$. Previously, F3.CD cells, immortalized human fetal NSCs expressing the therapeutic $\mathrm{CD}$ gene, led to a marked reduction in tumor burden and significantly prolonged the survival of brain tumor-bearing animals (5). In various animal models, the safety, feasibility and efficacy of NSCs to track invasive tumor cells and distant microtumor foci, and their ability to deliver therapeutic gene products to tumor cells have been confirmed. Thus, an effective 
A

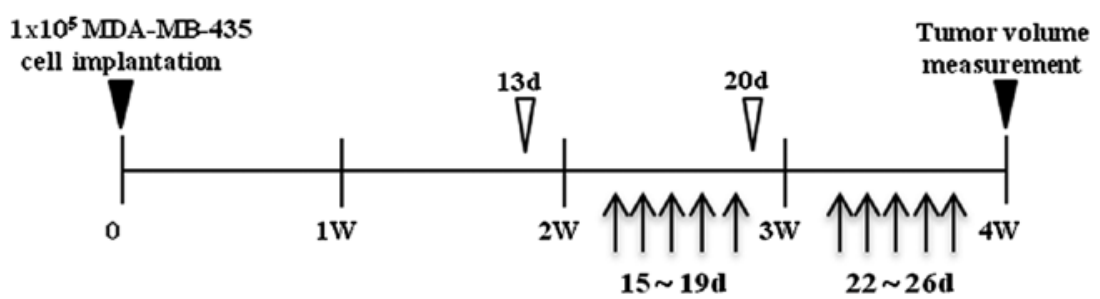

$\nabla$ : intracranial injection of $1 \times 10^{\delta} \mathrm{HB1}$.F3.CD (in $5 \mu \mathrm{lHBSS}$; GI and II) or

$5 \mu \mathrm{HBSS}$ (GIII and IV)-contralateral to the tumor cell implantation
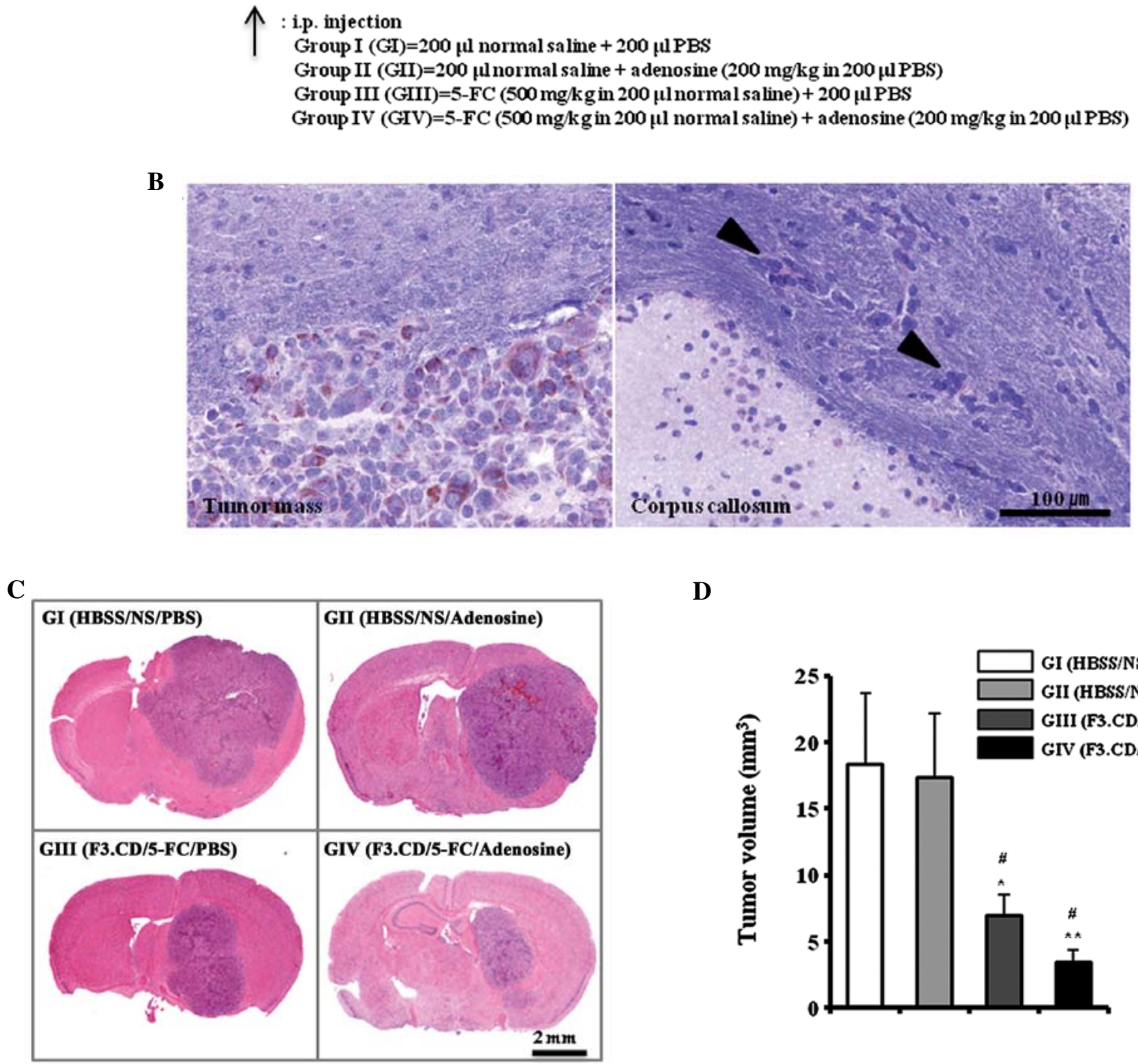

D

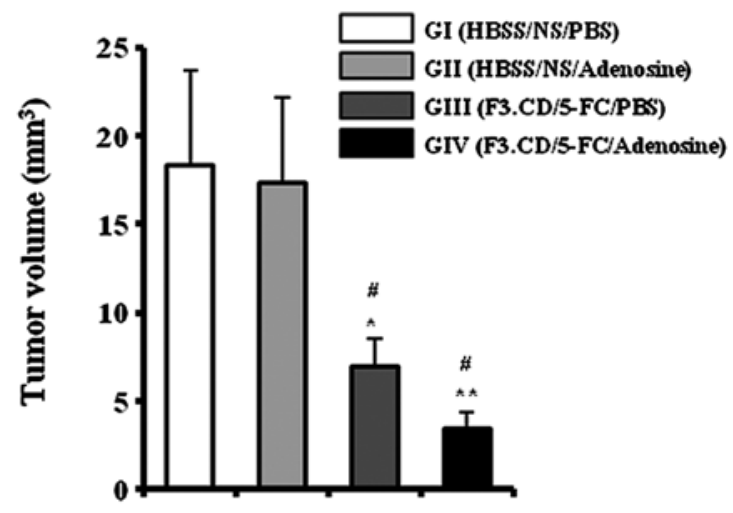

Figure 5. In vivo therapeutic effects of the F3.CD cells and 5-FC treatment against metastatic brain tumors were significantly increased by systemic addition of adenosine. (A) Experimental schedule was as illustrated. (B) F3.CD cells were injected into the brain contralateral to the tumor-bearing hemisphere. They were visualized by immunohistochemistry using an anti-CD antiserum. Arrowheads indicate CD-immunoreactive F3.CD cells in the corpus callosum. (C and D) Compared with Group III (F3.CD + 5-FC), systemic injection of adenosine (Group IV) resulted in a significantly reduced tumor volume. Bar, mean; error bars, SE; HBSS, Hanks' balanced salt solution; i.p., intraperitoneal; ${ }^{\mathrm{P}} \mathrm{P}<0.05 ;{ }^{* *} \mathrm{P}<0.01$ vs. Group I; ${ }^{"} \mathrm{P}<0.05$ vs. Group II.

antitumor modality overcoming the obstacles of current gene therapy strategies have been provided (8), leading to the FDA approval of the first human NSC clinical trial to treat glioma patients in 2010.

We previously reported this advantage on brain metastatic tumors using the same animal model as this study (5). The cytotoxic drug 5-FU is effective in the treatment of various primary tumors, but it suffers from the inability to penetrate across the BBB. However, 5-FC, the prodrug form of 5-FU, readily penetrates across the BBB into the brain parenchyma. $5-\mathrm{FC}$ is converted to 5-FU by $\mathrm{CD}$, an enzyme which is not encoded by the human genome. Therefore, if CD could be delivered to or locally expressed in MBTs, the prodrug would have great potential in the treatment of MBTs. Brain metastases are different from primary brain tumors such as gliomas and medulloblastomas since they originate from cells that do 
not reside in the brain. We showed that NSCs injected into the contralateral hemisphere migrate to breast tumor brain metastases. This suggests that breast tumor brain metastases attract NSCs.

In the present study, we tested the hypothesis that sufficient ribonucleoside could potentiate the tumor cell inhibitory effect of F3.CD and 5-FC treatment. 5-FU locally produced by F3.CD cells could be degraded by a cell detoxification system before it is converted to its toxic metabolites, 5-FU ribonucleoside (5-FUR) or 5-FU ribonucleotide $(9,10)$. The putative mechanism of ribonucleoside addition is that it would deviate the balance of the chemical reaction by the endogenous enzyme nucleoside phosphorylase to produce 5-FUR (Fig. 2). In case of no adenosine (ribonucleoside) supplementation, nucleoside phosphorylase uses endogenous ribose-1-phosphate. However, the concentration of 5-FUR produced from endogenous ribose-1-phosphate was minimal, and could not be detected in this study. In vitro and in vivo, survival and/or proliferation of tumor cells were inhibited more significantly by F3.CD and 5-FC when adenosine was supplied.

Simple adenosine or addition of another nucleoside could improve the antitumor activity of NSCs carrying the therapeutic gene CD. This supplementation method could be applied to other suicide gene therapies against various tumor types. To the best of our knowledge, this is the first report applying adenosine supplementation therapy to NSC-based gene therapy. Our demonstrated method may further increase therapeutic potential and thereby the clinical applicability of NSC-based gene therapy further.

\section{Acknowledgements}

This study was supported by a grant from the National R\&D Program for Cancer Control, Ministry of Health and Welfare, Republic of Korea (0820310) and by a grant of the Korea Health Technology R\&D Project, Ministry of Health and Welfare, Republic of Korea (A120446).

\section{References}

1. Patchell RA: The management of brain metastases. Cancer Treat Rev 29: 533-540, 2003.

2. Seol HJ, Jin J, Seong DH, Joo KM, Kang W, Yang H, Kim J, Shin CS, Kim Y, Kim KH, Kong DS, Lee JI, Aboody KS, Lee HJ, Kim SU and Nam DH: Genetically engineered human neural stem cells with rabbit carboxyl esterase can target brain metastasis from breast cancer. Cancer Lett 311: $152-159,2011$
3. Aboody KS, Najbauer J, Schmidt NO, Yang W, Wu JK, Zhuge Y, Przylecki W, Carroll R, Black PM and Perides G: Targeting of melanoma brain metastases using engineered neural stem/progenitor cells. Neuro Oncol 8: 119-126, 2006.

4. Schmidt NO, Przylecki W, Yang W, Ziu M, Teng Y, Kim SU, Black PM, Aboody KS and Carroll RS: Brain tumor tropism of transplanted human neural stem cells is induced by vascular endothelial growth factor. Neoplasia 7: 623-629, 2005.

5. Joo KM, Park IH, Shin JY, Jin J, Kang BG, Kim MH, Lee SJ, Jo MY, Kim SU and Nam DH: Human neural stem cells can target and deliver therapeutic genes to breast cancer brain metastases. Mol Ther 17: 570-575, 2009.

6. Ito S, Natsume A, Shimato S, Ohno M, Kato T, Chansakul P, Wakabayashi T and Kim SU: Human neural stem cells transduced with IFN-beta and cytosine deaminase genes intensify bystander effect in experimental glioma. Cancer Gene Ther 17: 299-306, 2010.

7. Lee SJ, Kim Y, Jo MY, Kim HS, Jin Y, Kim SU, Jin J, Joo KM and Nam DH: Combined treatment of tumor-tropic human neural stem cells containing the CD suicide gene effectively targets brain tumors provoking a mild immune response. Oncol Rep 25: 63-68, 2011.

8. Kim JH, Kim JY, Kim SU and Cho KG: Therapeutic effect of genetically modified human neural stem cells encoding cytosine deaminase on experimental glioma. Biochem Biophys Res Commun 417: 534-540, 2012.

9. Christensen CL, Zandi R, Gjetting T, Cramer F and Poulsen HS: Specifically targeted gene therapy for small-cell lung cancer. Expert Rev Anticancer Ther 9: 437-452, 2009.

10. Rogulski KR, Kim JH, Kim SH and Freytag SO: Glioma cells transduced with an Escherichia coli CD/HSV-1 TK fusion gene exhibit enhanced metabolic suicide and radiosensitivity. Hum Gene Ther 8: 73-85, 1997.

11. Christensen CL, Gjetting T, Poulsen TT, Cramer F, Roth JA and Poulsen HS: Targeted cytosine deaminase-uracil phosphoribosyl transferase suicide gene therapy induces small-cell lung cancerspecific cytotoxicity and tumor growth delay. Clin Cancer Res 16: 2308-2319, 2010.

12. Lee HJ, Kim KS, Kim EJ, Choi HB, Lee KH, Park IH, Ko Y, Jeong SW and Kim SU: Brain transplantation of immortalized human neural stem cells promotes functional recovery in mouse intracerebral hemorrhage stroke model. Stem Cells 25: 1204-1212, 2007.

13. Kim SU and de Vellis J: Stem cell-based cell therapy in neurological diseases: a review. J Neurosci Res 87: 2183-2200, 2009.

14. Seol HJ, Yoo HY, Jin J, Joo KM, Kim HS, Yoon SJ, Choi SH, Kim Y, Pyo HR, Lim DH, Kim W, Um HD, Kim JH, Lee JI and Nam DH: The expression of DNA damage checkpoint proteins and prognostic implication in metastatic brain tumors. Oncol Res 19: 381-390, 2011. 